\title{
Elaboración de un modelo de navegación tridimensional sobre el paisaje arqueológi- co de Jaén
}

Luis Ma Gutiérrez Soler *

Eva Ma Alcázar Hernández. ***

$M^{a}$ Angeles Royo Encarnación ****

Antonella Guidazzoli ******

Luigi Calori ******

\section{INFORMÁTICA Y ARQUEOLOGÍA EN JAÉN.}

Uno de los grandes avances de la arqueología durante los últimos años ha sido la incorporación de las posibilidades que ofrecen las ciencias matemáticas de la estadística y la informática, que son tenidas en cuenta de forma explícita como un campo más dentro de la interdisciplinareidad que forma parte de numerosos proyectos de investigación (RUIZ y MOLINOS, 1992), sin olvidar el interés que este tipo de propuestas despierta en el ámbito de la Administración, encargada de la tutela del Patrimonio.

Resultado de este interés creciente en el campo de la informática, con equipos técnicos cualificados, por parte de las Áreas de Prehistoria, Arqueología e Historia Medieval del Departamento de Territorio y Patrimonio Histórico de la Universidad de Jaén ha sido el desarrollo de un proyecto I+D entre el G.I.P.A.J. (Grupo de Investigación del Patrimonio Arqueológico de Jaén) y los Departamentos de
Ingeniería, Cartográfica, Geodésica y Fotogrametría y de Lenguajes y Sistemas Informáticos de la Escuela Politécnica de Jaén y la obtención de una subvención Icarus (GUTIÉRREZ y ALCÁZAR, 1995), incluida en el Programa de Capital Humano y Movilidad de la Comunidad Económica Europea para una estancia en el CINECA (Bolonia).

Formando parte del Proyecto "Arqueología y Ciencia Experimental para la recuperación y conservación del Patrimonio Arqueológico"' está en proceso de elaboración un Sistema de Información Geográfico (GIS) en el que está previsto que se incluya toda la documentación arqueológica del entorno de la ciudad de Jaén, abarcando un amplio territorio ${ }^{2}$ en el que quedaría contenida toda la información proporcionada por las numerosas excavaciones urbanas que se han venido desarrollando durante los últimos años en la ciudad de Jaén ${ }^{3}$, así como por todas las efectuadas en el marco de los Proyectos de Investigación que se llevan a cabo en La Campiña sobre el mundo Ibérico y sobre la

\footnotetext{
I Proyecto I+D incluido en el Plan Nacional de Investigación Científica y Desarrollo Tecnológico, financiado por el Ministerio de Educación y Ciencia.

2 Este territorio incluye 6 hojas cartográficas a escala I: I 0.000 con los números de referencia (947) I-I, I-2, 2-I, 2-2, 3- I y (926) 3-4.

3 Todo este importante volumen de información arqueológica generado por las intervenciones urbanas en la ciudad de Jaén está siendo elaborado informáticamente en el Proyecto "Estudio Arqueológico del Casco Urbano de Jaén", financiado por la Consejería de Cultura de la Junta de Andalucía, bajo la dirección de la arqueóloga Da Ma del Carmen Pérez Rodríguez desde I993 (ALCÁZAR, I994 y PÉREZ et alii, 1994). El objetivo último de este Proyecto es la creación de un Sistema de Información Arqueológica Urbana.
}

* Departamento de Territorio y Patrimonio histórico. Área de Prehistoria;

** Departamento de Territorio y Patrimonio histórico. Área de Historia Medieval

******ar Arqueóloga profesional;

***** Miembros del Consorcio Interuniversitario per la gestione del Centro di Calcolo Elettronico dell'Italia Nord-Orientale (CINECA) con sede en Bolonia 
etapa musulmana ${ }^{4}$, en los que se incluyen prospecciones, sondeos y excavaciones, especialmente en el oppidum de Puente Tablas y en el poblado del Cerro de Peñaflor respectivamente y por otras intervenciones financiadas en la zona por la Delegación Provincial de la Consejería de Cultura de Jaén.

Este ambicioso proyecto, una vez concluido, permitirá la organización en un Sistema de Información Geográfico de toda la documentación arqueológica disponible en la zona seleccionada en una amplia red, que permita la interconexión de múltiples bases de datos georeferenciados.

Paralelamente, hemos desarrollado una segunda línea de actuación en el campo del trabajo informático, que permitiera en un futuro vertebrarse con los resultados que hemos venido obteniendo en el proyecto I+D.

La oportunidad para llevar a efecto este segundo proyecto, como ya hemos dicho, ha venido de la mano de la concesión de una financiación Icarus, permitiéndonos acceder a los recursos técnicos y humanos que ponía a nuestro servicio el CINECA, cuyo Laboratorio de Visualización Científica, tiene una larga experiencia en proporcionar al cálculo prestaciones de modelización, control gráfico de simulaciones y síntesis de imágenes (CALORI y GUIDAZZOLI, 1993), contando con varias intervenciones en el campo de la arqueología.

La actividad de investigación interdisciplinar en campo arqueológico se ha desarrollado en el CINECA a partir del 1989-1990, en virtud de un acuerdo de colaboración científica entre el Centro di Calcolo y el Dipartimento de Scienze dell'Antichità de la Universidad "La Sapienza" de Roma. Desde entonces, la actividad científica se ha intensificado particularmente, implicándose otras instituciones de investigación como la Scuola Normale de Pisa (Topografia Antica) y el ISMEO, el Istituto italiano para el Medio y Extremo Oriente de Roma.
Actualmente están en curso de actuación en el CINECA el proyecto multimedia ARCTOS (Archeologia computazionale: metodologie de visualizzazione per la realizzazione di un sistema multimediale su un campione siciliano), en colaboración con la Scuola Normale Superiore de Pisa y la IBM SEMEA, y el ICARUS Project (Intensive Computing for Advanced Interdisciplinary Research of European Scientists), un programa de investigación financiado por la CEE que ve cómo interactúan en los campos arqueológico e informático varias universidades y centros de investigación europeos, como son el CNRS (Centre National de la Recherche Scientifique) de París, la Universidad de Jaén (España) y las Universidades de Essex y Cambridge (Gran Bretaña).

Las aplicaciones y las actividades de investigación de los diferentes institutos se diversifican en varios sectores, como la teledetección, los GIS, el tratamiento informático de las imágenes y la elaboración de modelos de simulación para el estudio de las dinámicas sociales y antropológicas del mundo antiguo. En este último campo son de particular interés las investigaciones sobre la simulación computerizada de la evolución de las sociedades del Bronce Antiguo (3200-I700 a.C.) en la península de Omán y sobre la evolución social del Paleolítico Superior en el SE de Europa.

En relación con el tratamiento informático y con la síntesis de imágenes es de destacar la elaboración de navegaciones sobre el terreno, resultado de una colaboración continuada del CINECA con el Doctor Maurizio Forte (1993 y 1994), debiendo citarse entre éstas, las navegaciones tridimensionales sobre el valle del Reno (Bolonia) en correspondencia con la ciudad etrusca de Marzabotto (siglos VI-IV a.C.), la navegación por el interior del asentamiento de la Edad del Bronce Medio-Reciente de la terramara de S. Rosa de Poviglio (Reggio Emilia) y recientemente el modelo reconstructivo de la rocca de Entella (Sicilia), área en la

\footnotetext{
4 Los Proyectos de Investigación financiados por la Junta de Andalucía a los que nos referimos son "El poblamiento ibérico en la Campiña de Jaén", dirigido por los Dres. Ruiz Rodríguez y Molinos Molinos y "El poblamiento hispano-musulmán en la Campiña de Jaén", dirigido por el Dr. Salvatierra Cuenca y por D. Juan Carlos Castillo Armenteros.
} 
que se superponen asentamientos desde la primera Edad del Hierro hasta el periodo medieval.

Es precisamente en este campo de actuación en el que queremos desarrollar nuestra exposición, teniendo como objetivo transmitir nuestra experiencia desde un doble planteamiento, que no sólo se limite a explicar el fundamento de nuestro modelo arqueológico, sino que también desarrolle detalladamente los aspectos metodológicos-técnicos que han sido utilizados para realizar dicho proyecto, incluyendo por tanto una breve reseña explicativa de qué es un Laboratorio de Visualización Científica, y de cuáles son sus objetivos y finalidades.

\section{INTRODUCCIÓN AL CONCEPTO DE VISUALIZACIÓN CIENTÍFICA: LA PER- CEPCIÓN Y COMPRENSIÓN DE LOS DATOS, LA INEVITABLE EVOLUCIÓN HACIA LA REALIDAD VIRTUAL.}

Antes de la llegada de los ordenadores, la visualización de los datos científicos se limitaba a representaciones de observaciones provenientes del mundo real.

La creciente disponibilidad de sistemas de cálculo automático, que permiten generar datos del mundo real a partir de modelos matemáticos, ha dado impulso a un nuevo desarrollo de las técnicas de "visualización de datos" que hace posible la representación visual de datos teóricos obtenidos a partir de la experimentación numérica.

La Visualización Científica consiste en la utilización de técnicas de computer graphics para el análisis de conjuntos de datos descriptivos de fenómenos científicos.

El nacimiento de esta disciplina se asocia normalmente a la publicación de un informe de 1987 a cargo del National Science Foundation's Adivisory Panel on Graphics, Image Processing and Workstations (McCORMICK et alii, 1987). Esta publicación definía y justificaba la necesidad del desarrollo de técnicas de Visualización
Científica a causa de la cada vez mayor velocidad en la producción de datos provenientes ya sea de instrumentos de adquisición (scanner, sensores), como de simulaciones en superordenadores.

El documento además subrayaba la importancia de la producción de animaciones para mejorar la comprensión de datos variables en el tiempo, es decir, para analizar la dinámica de los fenómenos en examen.

Actualmente se desarrollan numerosos seminarios y conferencias sobre el tema de la Visualización Científica, entre todas citamos la ACM's SIGGRAPH Conference. Por otra parte, están disponibles numerosos libros (NIELSON et alii, 1990) y revistas especializadas en el tema.

Las técnicas de Visualización Científica deben ayudar al investigador a comprender la estructura cualitativa del fenómeno analizado.

El usuario de Visualización Científica no debe sólo percibir, sino también comprender la naturaleza de la información presente en los datos analizados.

La percepción o la comprensión dependen de las capacidades perceptivas, de la cultura y de la experiencia individuales. Las tendencias actuales de la investigación en el campo de la Visualización Científica intentan profundizar la comprensión del proceso de visualización con el fin de poder utilizar conocimientos de psicología de la percepción, junto a técnicas de diseño gráfico, para intervenir en la programación de sistemas de visualización cada vez más eficaces.

La visualización puede ser considerada como un instrumento potente, algo más que un método de cálculo: es el proceso de transformación de la información en una forma perceptible. Esta actividad puede comprender también representaciones de tipo auditivo o táctil, consintiendo el desplazamiento del mecanismo de análisis del ámbito simbólicoreconstructivo a un dominio perceptivomotor (ANTINUCCl, 1993). 
La visualización final de los datos científicos debe por tanto consentir al investigador el individualizar estructuras escondidas, resaltando en poco tiempo el contenido informativo de los datos mismos.

Por otra parte, hoy muchas herramientas de software para la Visualización Científica y el Tratamiento Informático de Imágenes son sistemas de tipo dataflow (AVS, Explorer, $\mathrm{KHO}$ ROS). De esta forma el coste de la implementación de nuevas aproximaciones, es decir, la creación de nuevas primitivas de visualización, exige la programación de nuevos módulos.

Los objetivos de una sesión de Visualización Científica pueden ser varios y por lo tanto requerir aproximaciones diferentes. Por ejemplo, se puede hacer necesaria la exploración interactiva en tiempo real de datos o bien una representación cuidada del fenómeno, o en fin, puede ser realizada una representación de los datos con objetivos didácticos.

Cada aproximación implica diferentes equilibrios entre diversas propiedades de la representación, como son la interactividad o la exactitud de los algoritmos. Las cada vez mayores prestaciones de los sistemas hardware harán más simples la integración de tales propiedades, incluso si simulaciones que produjeran enormes cantidades de datos del orden de los terabyte, implementadas sobre sistemas de cálculo masivamente paralelos, llegaran a ser cada vez más comunes, haciendo dificultoso el acceso interactivo a conjunto de datos tan amplios, siendo cada vez más necesaria la utilización de interface de tipo Realidad Virtual (MONTEFUSCO, 1994 y CRUZ-NEIRA et alii, 1993).

\section{LA EXPLORACIÓN INTERACTIVA DE LOS DATOS.}

El número cada vez mayor de representaciones de datos variables en el espacio y en el tiempo aconseja el uso de una herramienta de exploración interactiva. Estructuras tridimensionales complejas, comunes por ejemplo en el ámbito de la medicina, estructura de la materia, ciencias de la tierra y ambientales
(GIS), pueden dar lugar a ambigüedades si se representan sobre un espacio bidimensional.

Las interfaces de tipo Realidad Virtual preven espacios tridimensionales y dispositivos de input que ofrecen al usuario la experiencia de la inmersión en un espacio tridimensional en el que las estructuras pueden ser visualizadas en el espacio, de forma no ambigua.

El objetivo de este tipo de ambientes virtuales será el de proporcionar una interface intuitiva al ambiente de cálculo, presentando al investigador un objeto virtual para estudiar en vez de una banal representación bidimensional. En el caso de estructuras tridimensionales complejas, la utilización de técnicas de control virtual posibilitará al investigador cambiar rápidamente los datos a representar y los lugares de la visualización.

\section{EL PAPEL DE UN LABORATORIO DE VISUALIZACIÓN CIENTÍFICA. LA EXPE- RIENCIA DEL CINECA. HACIA LA TELE- VIRTUALIDAD.}

El Laboratorio de Visualización Científica del CINECA (ViscLab) (Fig. I), ofrece el uso de estaciones de trabajo y software dedicados a la visualización $2 D$ y $3 D$ integrados en red Ethernet en un ambiente de superordenadores (CRAY C94/2I 28 y MPP CRAY T3D/64) además del aparataje necesario para la producción de diapositivas y vídeos. En particular se dispone de una decena de ambientes de trabajo gráfico, entre los cuales se encuentra una Silicon Graphics Indigo, dispositivos de output sobre papel con tecnología laser, un sistema (FOCUS) para la producción de fotos y diapositivas y un sistema de videoregistración ACCOM digital, accesible vía red Internet como nodo TCPIP. Los vídeos maestros son realizados en formato Pal BETACAM.

Además se dispone de diversos tipos de software para la Visualización Científica y el tratamiento informático de las imágenes como: AVS, PV-WAVF, Khoros, NCSA-Tools, MPGS, ERDAS, Grass. Han sido desarrollados códigos ad hoc para aplicaciones concretas y para la gestión remota de los dispositivos 
vídeo. La presencia contemporánea de un ambiente de supercálculo y de Visualización Científica, integrado en red Internet, constituyen un ambiente de investigación particularmente potente y versátil.

En estos años se han producido visualizaciones en diversos sectores aplicativos: astrofísica, medicina, medioambiente, química computacional, oceanografía, termofluidodinámica, análisis estructural, ciencia de los materiales, física del estado sólido, gráfica computacional. En particular se han conducido experiencias de visualización en el campo arqueológico y se han registrado vídeos que reportan experiencias de "navegación sobre el paisaje".

Las experiencias actuales de desarrollo de instrumentos y servicios para la navegación en el espacio "virtual" de Internet (ver Software MOSAIC NCSA) dejan entrever futuros escenarios, en los que la disponibilidad de redes telemáticas cada vez más veloces hará posible utilizar espacios virtuales de colaboración entre investigadores para la compartimentación y el análisis de los datos, permitiéndoles intercambiar en este nuevo ambiente ideas, descubrimientos, experiencias.

\section{UNA EXPERIENCIA DE NAVEGA- CIÓN SOBRE EL PAISAJE ARQUEO- LÓGICO DE JAÉN.}

\section{ELABORACIÓN DEL MODELO ARQUEOLÓGICO.}

Antes de continuar exponiendo nuestros objetivos, querríamos hacer nuestras las palabras del Doctor M. Forte por considerarlas de un alto contenido aclaratorio respecto de la potencialidad del método para avanzar en el campo de la investigación arqueológica: "La possibilità di visualizzare al calcolatore il paesaggio archeologico come un modello tridimensionale fotorealistico, non grafico, ma pienamente rappresentativo della realtà fisica, ci restituisce un modello virtuale, uno spazio eidetico, in cui possiamo muoverci liberamente, navigando attraverso qualunque prospettiva, anche impossibile" (Forte, 1993).
La posibilidad de reconstruir en laboratorio el paisaje arqueológico a una escala reducida, permite una navegación de tipo intersites o intra-sites, en la que es posible organizar múltiples recorridos y elegir cualquier perspectiva del territorio, teniendo tan sólo como límite la definición de la imagen digital, o lo que es lo mismo, la unidad de medida de resolución visual de un pixel (Forte y Guidazzoli, 1992).

En nuestro caso, la zona elegida para llevar a cabo esta primera experiencia de navegación sobre un paisaje arqueológico ha sido el territorio que media entre la ciudad de Jaén y el oppidum ibérico de la Plaza de Armas de Puente Tablas, distantes entre sí algo más de 4 kilómetros en línea recta, que vendría a coincidir aproximadamente con una de las dos diagonales que pueden trazarse en el área sobre la que hemos desarrollado nuestro modelo.

La elección de estos límites territoriales viene motivada por el interés de continuar profundizando en el desarrollo de nuevas aplicaciones informáticas en la misma zona arqueológica sobre la que se está trabajando desde el proyecto I+D y por la posibilidad de articular un discurso de gran validez desde el campo de la investigación y de la protección y tutela del Patrimonio Arqueológico.

Desde el campo de la investigación el principal objetivo ha sido mostrar a través de la imagen la evolución del poblamiento sobre el territorio por horizontes culturales sucesivos, desde época prehistórica hasta el periodo medieval cristiano y simular virtualmente en el modelo tridimensional las relaciones que el poblamiento establece en cada uno de esos horizontes con el medio circundante, aunque deteniéndonos especialmente en mostrar el paisaje arqueológico del oppidum ibérico de Puente Tablas y en la compleja relación de conflicto territorial que debió establecer con el oppidum del olivar de Santa Catalina (Jaén), que recientemente está siendo fruto de una revisión historiográfica (SERRANO et alii, en prensa) a la luz de las nuevas secuencias estratigráficas obtenidas en la ciudad de Jaén y en el propio cerro de Santa Catalina (CASTILLO y CANO, 1994). 
Centrándonos en el caso concreto de Puente Tablas, la navegación a través del valle del río Guadalbullón hasta alcanzar el yacimiento nos permite comprender de una forma rápida y precisa la ubicación elegida para el emplazamiento del oppidum en relación a su entorno, ocupando una meseta que se levanta directamente sobre el río y que permite una fácil defensa y el control del territorio económica y políticamente dependientes, mientras que un acercamiento progresivo al asentamiento, siguiendo una navegación de tipo intra-site, nos permite sobrevolarlo y reconocer de esta forma la intervención arqueológica que se ha llevado a cabo sobre este yacimiento.

En relación al resto de asentamientos, su distribución por horizontes cronológicos permite una navegación de tipo inter-sites por el entorno arqueológico de Jaén, en la que es posible analizar la relación espacial que se establece entre dichos yacimientos, facilitando la elaboración de hipótesis históricas sobre la distribución del poblamiento, sobre la articulación de las redes de tránsito e intercambio, sobre la organización de las áreas de control territorial y, en suma, sobre la jerarquización de los asentamientos, sin olvidar el carácter fuertemente predictivo que ofrece el sistema para el descubrimiento de nuevos yacimientos en áreas aún no prospectadas.

Desde el punto de vista de la protección y tutela del Patrimonio Arqueológico esta navegación sobre el paisaje nos ha permitido visualizar sobre un modelo tridimensional los resultados de las últimas campañas de prospección realizadas en los terrenos recalificados como urbanizables en el entorno de la ciudad de Jaén (ZAFRA et alii, inédito), mostrando además, de una forma eficaz el peligro que corren numerosos yacimientos ante el inminente desarrollo de los planes de urbanización de las áreas más cercanas al núcleo urbano de la ciudad de Jaén.

Aparte de las posibilidades que este tipo de visualizaciones en movimiento ofrece para ser utilizado como documento administrativo, para definir estrategias de planifica- ción territorial con las que garantizar la protección y tutela de nuestro Patrimonio Arqueológico, no debemos olvidar un aspecto de gran importancia, el poder de difusión de este tipo de soporte, sobre todo por lo que respecta a las relaciones que pueden establecerse entre cada uno de los yacimientos y el paisaje de su entorno, tanto por lo que respecta a la estructura geomorfológica de la unidad geográfica en la que se emplaza como respecto al paisaje, aunque en ambos casos dichas aproximaciones necesitan un comentario crítico a la luz de las lecturas de la dinámica de degradación del paisaje y de las curvas polínicas y de los análisis de fauna llevados a cabo en el interior de cada yacimiento, que nos permita intentar reconstruir cómo debió evolucionar la relación hombremedio en cada una de las fases históricas definidas arqueológicamente.

\section{TRATAMIENTO INFORMÁTICO.}

Las fases principales de adquisición, organización y gestión de los datos y de la imagen para la elaboración de un modelo tridimensional por ordenador que permita una navegación sobre el paisaje arqueológico como la que hemos llevado a cabo son las siguientes:

- Adquisición de datos altimétricos georeferenciados (cartografía o relieve taquimétrico computerizado efectuado directamente sobre el terreno).

- Conversión de datos altimétricos en un Modelo Digital del Terreno.

- Adquisición y conversión digital de los datos teledetectados o de las tomas aéreas del área de interés.

-Eventual tratamiento informático de la imagen aérea o satélite digitalizada a fin de resaltar informaciones de particular interés.

- Eventual rectificación de la fotografía o de las tomas aéreas y alineamiento con el modelo altimétrico (sincronización de la imagen al Modelo Digital del Terreno). 
- Aplicación de la técnica texture mapping (integración de las imágenes digitales con su correspondiente modelo altimétrico) de la imagen aérea o satélite digital.

- Adquisición en formato vectorial de otros elementos o informaciones útiles para la elaboración (capas).

- Navegación virtual interactiva al interior del modelo reconstruido, producción de vídeos y diapositivas con un fin ilustrativo y didáctico.

\section{ADQUISICIÓN Y GENERACIÓN DEL DTM DEL ENTORNO GEOGRÁFICO DE JAÉN.}

Utilizando AutoCAD 12 ha sido preparado un archivo de tipo vectorial dxf, digitalizando las curvas de nivel cada 10 metros. Posteriormente fueron adquiridas otras capas de información con la digitalización de algunas carreteras y de los yacimientos arqueológicos, útiles para elaboraciones sucesivas.

\section{LECTURA Y DEFINICIÓN DE LA IMAGEN SATÉLITE UTILIZADA.}

La imagen utilizada para la reconstrucción del paisaje arqueológico de la zona de Jaén es una imagen satélite SPOT, disponible en formato CDROM, proporcionada por TELECOM ITALIA SPA, división SPAZIO. Es de tipo pancromático con una banda espectral, visualizable por lo tanto en niveles de gris y sobre ella ya se habían efectuado algunas correcciones geométricas y de tipo radiométrico teniendo en cuenta las características de la toma y del sistema satélite.

La imagen completa, de la cual se ha tomado la porción que recogía el área de interés, consta de 6010 líneas, mientras el número de pixel por línea es igual a 6590, que hacen un total de $8640 * 60 \mathrm{II}$ pixel, comprendiendo también los pixel negros de relleno (cerca de $52 \mathrm{Mb}$ de raw data).

La localización del centro de la escena de la imagen es la siguiente:
Latitud N037050' | 4"

Longitud W003036'47"

Número de pixels: 3266

Número de líneas: 3005

Cada pixel representa una zona cuadrada del terreno de una dimensión fija de 20 metros de lado, teniendo la imagen completa una dimensión fija de 60 kilómetros en dirección Norte-Sur.

En formato bw (blanco y negro) run lenght SGl la imagen ocupa cerca de $37 \mathrm{MB}$.

Ha sido además posible recortar y visualizar la zona de interés utilizando las herramientas scope y subimg sobre SGI Challange $L$ (sirio.cineca.it) y visualizándola en una estación de trabajo Indigo SGI (indigo.cineca.it).

La subimagen final de la zona de interés es igual a 1280 (xsize) 866 (ysize) pixels.

\section{ELABORACIÓN DEL DTM Y DE LA IMA- GEN DIGITAL MEDIANTE EL SOFTWARE GIS GRASS}

El software GRASS es un software de dominio público, por lo que se puede adquirir en su totalidad vía red sin costes adicionales (vía ftp anonymous a max.cecer.army.mil) y está en grado de realizar tratamiento informático de imágenes y sistemas de información geográfica.

Está escrito en lenguaje C y funciona en ambiente operativo Unix. Originariamente fue proyectado y desarrollado para asistencia a la gestión del territorio en instalaciones militares y actualmente es ampliamente utilizado en una vasta serie de aplicaciones en los Estados Unidos, que van desde el control y seguimiento geológicos hasta la gestión de los parques nacionales.

GRASS es un GIS compuesto por módulos tanto para la elaboración vectorial como para el análisis y la elaboración de imágenes raster. A estas prestaciones se añade la facultad de intercambiar datos con database, informaciones cartográficas y vectoriales, fotos aéreas, imágenes satélite y en general, todas las informaciones que se pueden obtener sobre un lugar. 
En el caso del estudio del entorno geográfico de Jaén, y trabajando con Grass, se ha definido la zona de interés en coordenadas UTM, manteniéndose dicha definición durante todo el procedimiento.

El archivo en formato $\mathrm{d} x f$ generado por AutoCAD ha sido importado a Grass, utilizando la rutina vin.dxf (GRASS Reference Manual), al igual que la subimagen escogida (vin.ras). Esta última ha sido sometida a un proceso de mejoramiento digital del contraste para hacerla más legible a la hora de efectuar la navegación sobre el territorio (r.map.cale).

La imagen ha sido rectificada utilizando las rutinas i.points e i.rectify. La primera función ha consentido marcar en la imagen utilizando un ratón, puntos conocidos, y por lo tanto insertar en input las correspondientes coordenadas geográficas creando de esta manera una matriz de coeficientes de transformación, que serán utilizados por el módulo i.rectify que opera una transformación lineal afín para obtener la rectificación de la imagen.

El Modelo Digital del Terreno (que en origen se encuentra en formato vectorial), siempre en ambiente Grass, ha sido rasterizado para permitir la creación de una superficie gracias a la función r.surfidw que calcula una interpolación de las pendientes de la superficie a través de una aproximación numérica basada sobre los valores de los puntos próximos.

La sincronización del Modelo Digital del Terreno con la imagen rectificada y la navegación en el territorio han sido realizados utilizando SG3d, un módulo de Grass en grado de visualizar datos de superficie en tres dimensiones que, como recurso hadware, exige un Z-buffer y una gráfica a 24 bit. SG3d necesita en input un archivo raster para utilizar como modelo digital de elevación y otro archivo raster que actúa de textura del precedente. Mientras el file que recoge las informaciones altimétricas, usado como modelo de elevación, reproduce de forma realística las superficies, el usuario puede seleccionar también otros tipos de datos representables en la dimensión vertical (por ejemplo las clases de materiales de un yacimiento arqueológico). La mayoría de los datos de tipo continuo (no discreto) puede ser significativamente visualizados. El módulo SG3d ha sido modificado para obtener una mejor calidad del vídeo de navegación en el territorio y para acelerar los tiempos de ejecución, desactivando el doble buffer de tal forma que dicho módulo produjese en salida los archivos en formato vídeo digital yuv, preparados para ser enviados al videoregistrador digital Accom en red TCPIP pilotable en modo remoto a través de los procedimientos desarrollados en el Laboratorio de Visualización Científica del CINECA.

Por último, se ha realizado una animación sobre soporte vídeo Betacam SP (25 frames por segundo) de algunos minutos de duración que propone algunos recorridos de navegación, evidenciando también las capas vectoriales de los yacimientos arqueológicos. Han sido además producidas diapositivas utilizando el dispositivo FOCUS para documentar todo el proceso y algunas vistas particularmente significativas del paisaje reconstruido.

\section{CONCLUSIONES}

La integración de datos altimétricos adquiridos cartográficamente o directamente captados sobre el terreno, con las fotografías aéreas o los datos teledetectados correspondientes a las áreas de exploración, constituyen el modelo fundamental para análisis de tipo territorial y para la reconstrucción de paisajes arqueológicos. Partiendo de estos datos podemos efectivamente emprender investigaciones de tipo intra-site, es decir, dentro de un asentamiento arqueológico, o de tipo inter-sites, dirigido al estudio de varios asentamientos que constituyen un sistema articulado de ocupación del territorio. De este modo es posible realizar cartas arqueológicas tridimensionales, aplicaciones de tipo GIS 3D, incluso con análisis estadístico-espaciales, realizando para cada aplicación navegaciones virtuales interactivas. 
La realización de modelos extremadamente complejos de paisajes arqueológicos, pero también de áreas aún no exploradas, podrá producir resultados de carácter fuertemente predictivo para la planificación de las futuras investigaciones sobre el terreno, identificando yacimientos arqueológicos no indagados, geomorfologías, reconstrucciones ambientales y paleoambientales. En este sentido el yacimiento arqueológico encontrará plena representación en la evolución morfológica y artificial del paisaje, incluyéndose dentro del antiguo sistema topográfico al que pertenece y como tal interpretado.

La perspectiva de trabajar con sistemas de cálculo siempre más potentes, hace que el escenario que logramos imaginar para la ciencia arqueológica computacional de los próximos años sea el de una total interactividad virtual de la investigación. La reconstrucción y elaboración de modelos complejos, ya sean paisajes arqueológicos o yacimientos concretos, será accesible, via red, para institutos de investigación e investigadores particulares, que podrán interactuar contemporáneamente y en tiempo real, sobre un modelo de investigación, emprendiendo diferentes caminos de acceso y de exploración, y comparando los datos sobre la base de la propia experiencia

\section{BIBLIOGRAFÍA.}

ALCÁZAR, E. (ep): "La Carta de Riesgo de la Ciudad", Códice, $n^{\circ} 9$.

ALCÁZAR, E. (1994): "Propuesta metodológica para la creación de un Sistema de Información Arqueológica de la ciudad", Revista de Arqueología y Teritorio Medieval. Número I. Jaén, pp. 209-2I5.

ANTINUCCI, F. (1993): "Dalla mente simbolica a quella sensomotoria", Realtà Virtuale, pp. I-6.

AVS Programmer manuals Advanced System Inc.

CALORI, L. y GUIDAZZOLI, A. (1993): "Esperienze di visualizzazione scientifica al CINECA", Scienza e Supercalcolo al CINECA, Rapporto 1993. Bolonia, pp. 306-308.

CASTILLO, J.L. y CANO, J. (inédita): "Memoria de la diagnosis arqueológica en la muralla norte de Jaén".
CRUZ-NEIRA, C; LEIGH, J.; BARNES, C;; COHEN, S.M.; DAS, S.; ENGELMANN, R; HUDSON, R.; PAPKA, M.; ROY, T.; SIEGEL, L.; VASILAKIS, C.; DEFANTI, T.A. y SANDIN, D.J. (1993): "Scientists in Wonderland: A Report on Visualization Applications in the CAVE Virtual Reality Environment", IEEE 1993 Symposium on Research Frontiers in Virtual Reality, pp. 55-66.

GRASS Reference Manual U.S. Army CERL, Spring '93.

GUTIÉRREZ, L.M. y ALCÁZAR, E. (1995): "Image processing for archaeological uses", Flying High. Número 3.

FORTE, M. (1993): "Il paesaggio archeologico al calcolatore", Le Scienze. Número 298, pp. 46-54.

FORTE, M. (1994): "Archeologia del terzo millennio. Padroni del passato", Virtual. Número 10. Milán, pp. 42-46.

McCORMICK, B.H.; DEFANTI, T.A. y BROWN, M.D. (1987): "Visualization in Scientific Computing" eds., Computer Graphics. Volumen 2I, número 6.

MONTEFUSCO, D. (1994): "Realtà Virtuales: tecnologia e applicazioni", Atti European Conference on Virtual Reality in Education, Training and Disability, pp. 15-35.

NIELSON, G.M.; SHIVER, B. y ROSENBLUM, L.J. (1990): Visualization in Scientific Computing, eds., IEEE Computer Society Press, Los Alamitos, Clif., Ag.

PÉREZ, Ma.C. (inédito): "Estudio Arqueológico del Casco Urbano de Jaén. Un proyecto de futuro", Ponencia a las VII Jornadas de Arqueología Andaluza. Córdoba, 27-29 de Noviembre de 1994.

PÉREZ, Ma.C; DE LA CASA, J. y AGUILAR, J.D. (1994): "Política general de protección y estrategias de intervención en el casco histórico de la ciudad de Jaén", Boletín del Instituto de Estudios Giennenses. Volumen 152. Jaén., pp. 239-249.

RUIZ, A. y MOLINOS, M. (1992): "Proyecto: Poblamiento Ibérico en La Campiña de Jaén", Investigaciones Arqueológicas en Andalucía 1985-1992, Huelva 1993, pp. 543-578.

RUIZ, A. y MOLINOS, M. (1993): Los Iberos. Análisis arqueológico de un proceso histórico. Crítica.

SALVATIERRA, V.; ALCÁZAR, E. y PÉREZ, M.C. (1993): "La prospección en arqueología medieval: el territorio y la ciudad", Revista de la Facultad de Humanidades. Volumen II, tomo 2. Jaén, pp. I I5- 124.

SERRANO, J.L; JIMÉNEZ, Y. y ZAFRA, J. (en prensa): "Aurgi: un municipio romano desde la arqueología urbana".

ZAFRA, J.; SERRANO, J.L.; ROYO, M.A. y PÉREZ, M.C. (inédito): "Estudio Arqueológico del casco urbano de Jaén. Memoria de la prospección arqueológica superficial en el suelo urbanizable del término municipal de Jaén". 


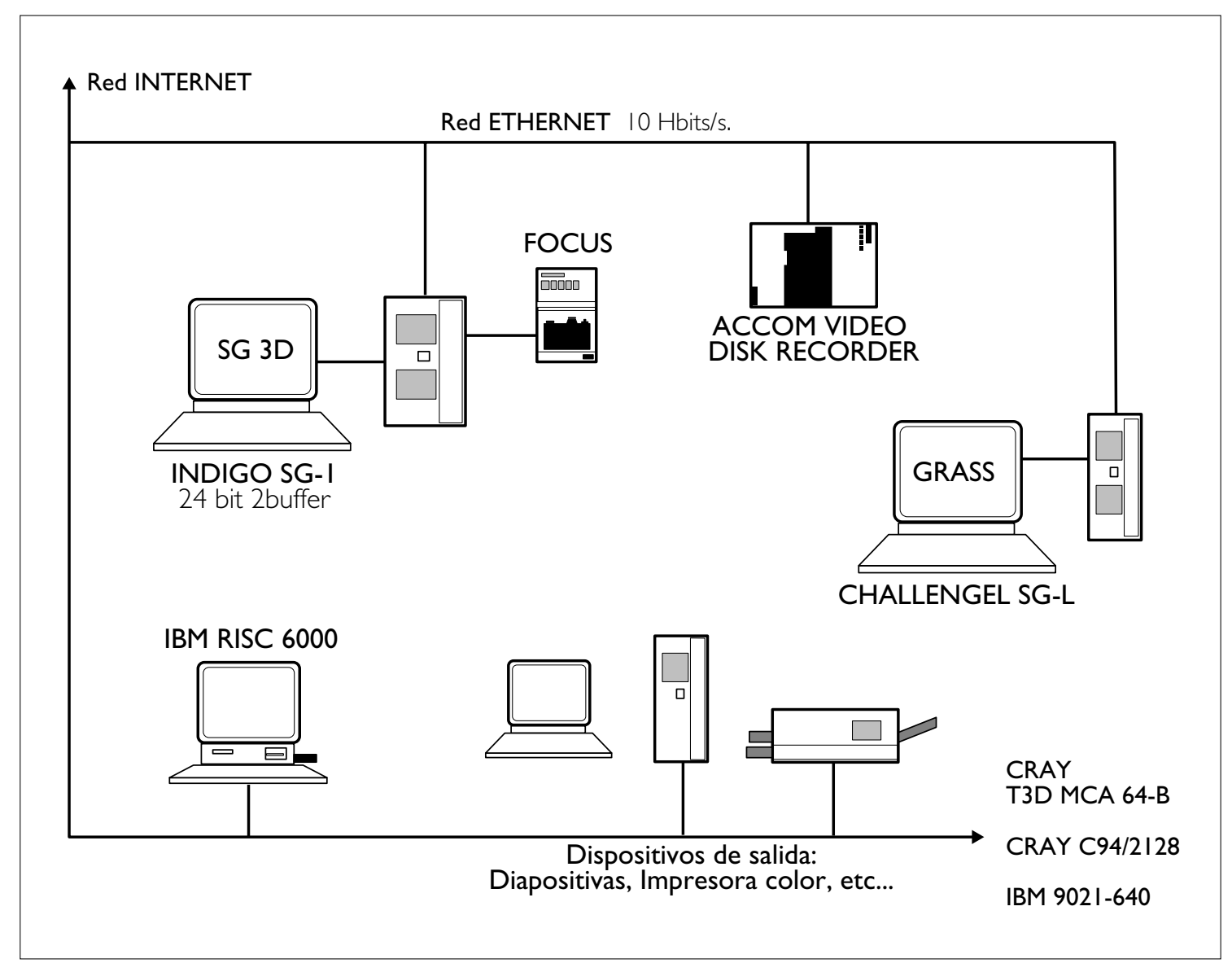

Fig. I. Entorno informático del Laboratorio de Visualización Científica del CINECA

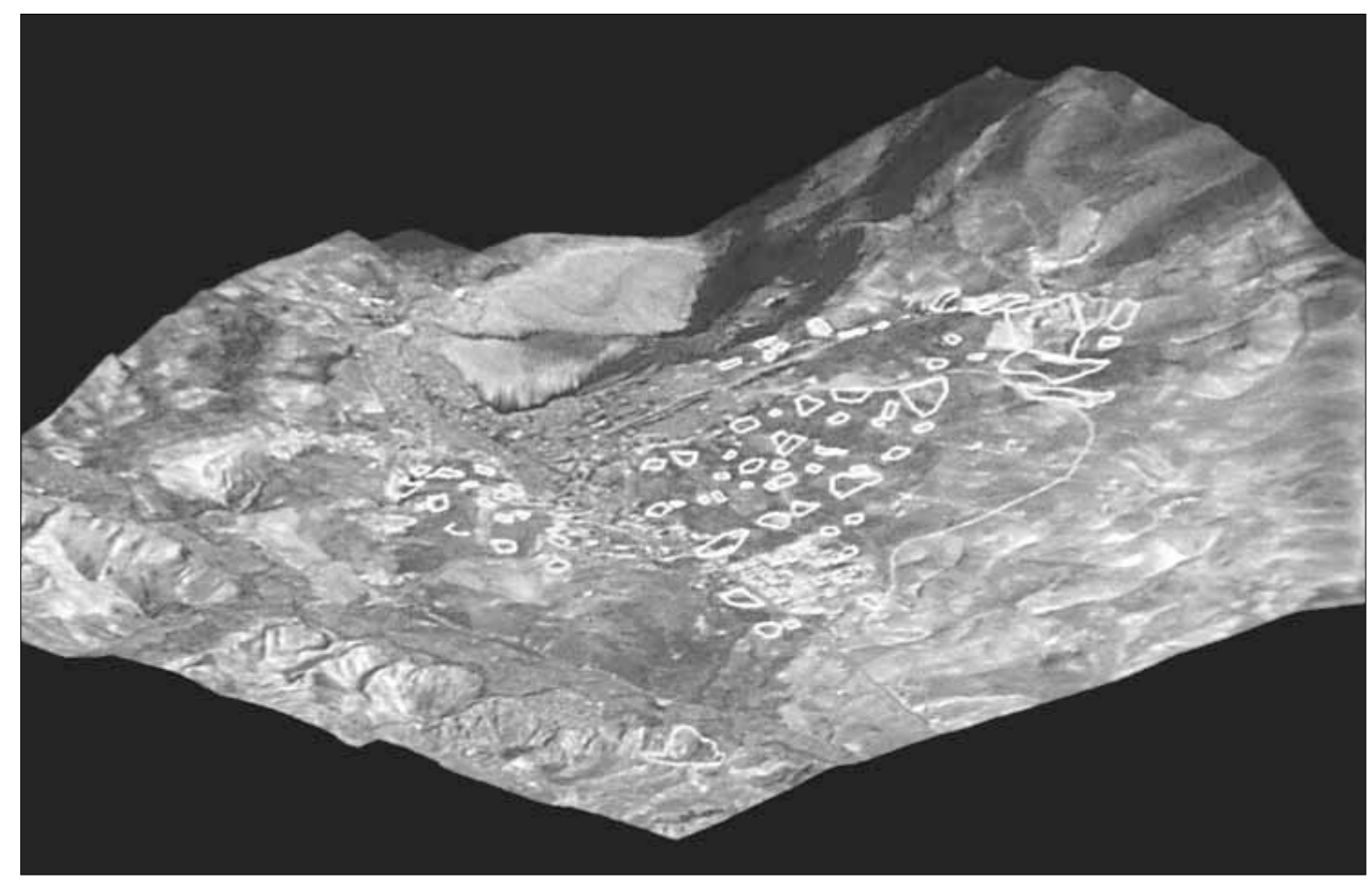

Fig. 2. Vista general del modelo tridimensional con la superposición de la capa vectorial que contiene la delimitación de los yacimientos 


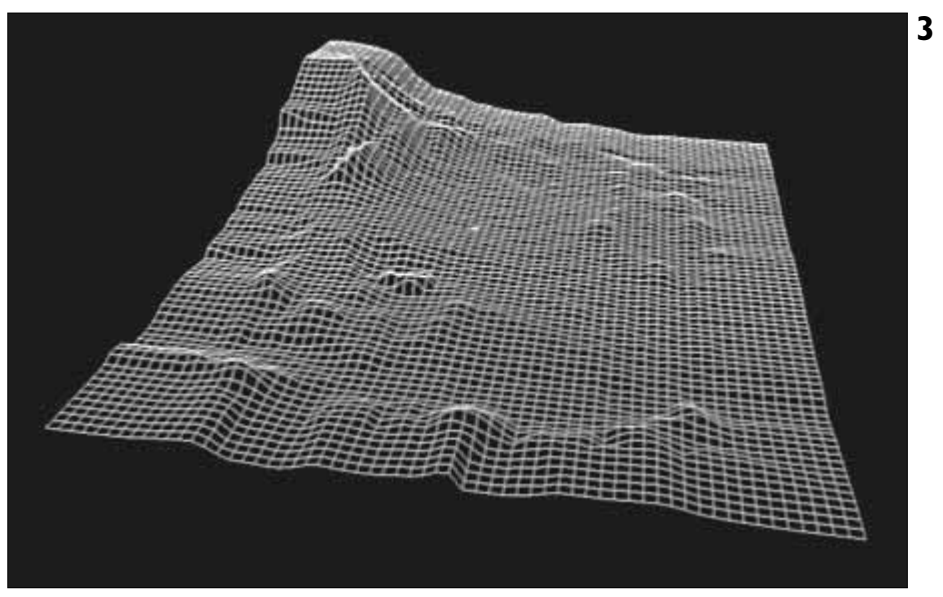

Fig. 3. Modelo digital del terreno

Fig. 4. Modelo altimétrico con seudo- color (para la impresión se ha traducido el espectro de color en niveles de gris).

Fig. 5. Tratamiento informático del modelo tridimensional, evidenciando el microrrelieve del terreno en base al grado de pendiente del suelo
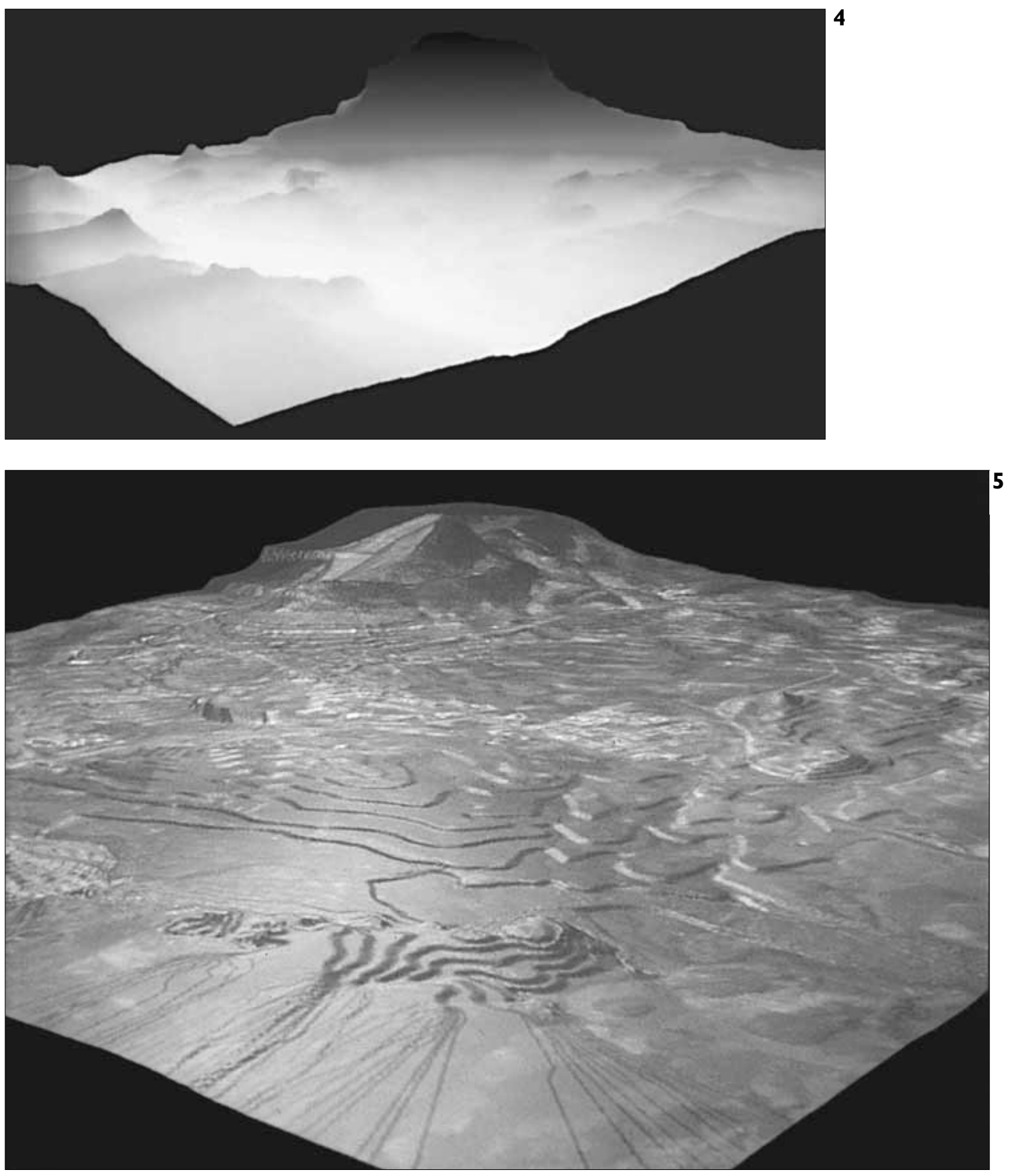